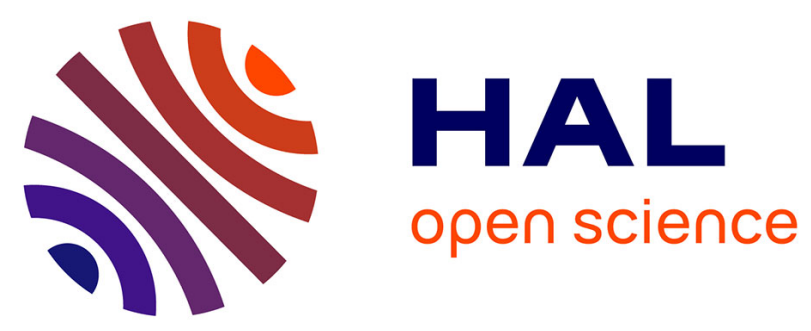

\title{
Design and performance evaluation of a DSP visible light communication receiver
}

Alin Cailean, Barthélemy Cagneau, Luc Chassagne, Valentin Popa, Mihai Dimian

\section{- To cite this version:}

Alin Cailean, Barthélemy Cagneau, Luc Chassagne, Valentin Popa, Mihai Dimian. Design and performance evaluation of a DSP visible light communication receiver. 21th IEEE Symposium on Communications and Vehicular Technology, SCVT, 2014, Delft, Netherlands. pp.30-34. hal-01207171

\section{HAL Id: hal-01207171 \\ https://hal.science/hal-01207171}

Submitted on 1 Oct 2015

HAL is a multi-disciplinary open access archive for the deposit and dissemination of scientific research documents, whether they are published or not. The documents may come from teaching and research institutions in France or abroad, or from public or private research centers.
L'archive ouverte pluridisciplinaire HAL, est destinée au dépôt et à la diffusion de documents scientifiques de niveau recherche, publiés ou non, émanant des établissements d'enseignement et de recherche français ou étrangers, des laboratoires publics ou privés. 


\section{Design and performance evaluation of a DSP visible light communication receiver}

\author{
Alin-Mihai Cailean ${ }^{1,2, *}$, Barthélemy Cagneau ${ }^{1}$, Luc \\ Chassagne ${ }^{1}$ \\ ${ }^{1}$ Université de Versailles, \\ Laboratoire d'ingénierie des systèmes de Versailles \\ Vélizy-Villacoublay, France \\ alinc@eed.usv.ro
}

\author{
Valentin Popa ${ }^{2}$ and Mihai Dimian ${ }^{2}$ \\ ${ }^{2}$ Stefan cel Mare University, Department of Computers, \\ Electronics and Automation \\ Suceava, Romania
}

\begin{abstract}
This paper proposes a new architecture for outdoor low data rate visible light communication applications. Considering the performances of the digital filtering, the proposed architecture considers the usage of digital signal processing (DSP) as an alternative to the analog signal treatment. The key aspects related with the implementation of the VLC receiver are discussed and motivated through simulations. The simulation results demonstrate the performances of the proposed VLC receiver for different noise levels.
\end{abstract}

Keywords- digital signal processing; IEEE 802.15.7; intensity modulation; optical communications; visible light communications.

\section{INTRODUCTION}

In the last years we can notice a strong demand for wireless access technologies in multiple fields. Up until few years, the wireless communications were clearly dominated by Radio Frequency (RF) communications, with no perspective for a different strong contra candidate. However, in the recent years, with the advancements in the solid state lighting technology, high performances Light Emitting Diodes (LEDs), capable of rapid switching, enabled Visible Light Communications (VLC). VLC is an emerging wireless communication technology which uses the visible light spectrum, from $380 \mathrm{~nm}$ to $780 \mathrm{~nm}$, as a communication medium. Expanding on such a wide spectrum offers VLC the potential to achieve very high data rates, as in [1]. Unlike RF based wireless communications, VLC is safe for human health and for high precision instruments, having the possibility to be used in RF-restricted areas such as airplanes or hospital intensive care units, as in [2]. Since in VLC the light is amplitude modulated by rapid switching LEDs, its development is sustained by the expansions of the solid state lighting industry, which is now able to produce high quality LEDs at a low price. Under these circumstances, LED lighting sources are meant to gradually replace the classical lighting sources [3, 4], making VLC a ubiquitous technology. LED lighting has begun to be used in the field of transportation, in vehicle lighting systems and in traffic infrastructures, such as traffic lights or street lighting systems [5]. In the area of transportation, wireless communications are required towards the development of the communication-based safety applications, as part of the Intelligent Transportation System (ITS). Here, VLC can be used for Vehicle to Vehicle (V2V) and Infrastructure to Vehicle (I2V/V2I) communications, but also for the cooperation between the two, as demonstrated in $[6,7]$, in [8 11], respectively in [12]. This way, vehicles are able to share traffic safety information (e.g. velocity, brake, direction, mechanical state, etc.) that can substantially increase the traffic safety. Such a scenario is illustrated in Fig. 1. The traffic light broadcasts the traffic safety information, containing its location, state, the time before next change and other information about the speed limits and routes to follow. The van receives this data and forwards it to the following vehicle using its tail lights.

In ITS, VLC can be used as an alternative and/or a complement for the radio frequency dedicated short range communications (DSRC) [13]. Due to the relatively limited communication distances $(10-80$ meters $), \mathrm{VLC}$ is suitable mostly in high density traffic, where a large number of nodes are located within a limited area [14]. Moreover, when these conditions are fulfilled, the usage of the DSRC is rather questionable. The close by vehicles will cause mutual interferences that will increase the latencies and will have a negative effect on the packet delivery ratio $[15,16]$.

Some main reasons for the relatively limited range of the VLC systems are signal degradation and the strong noise interferences that affect the signal to noise ratio (SNR). The SNR can be enhanced by using optical lens, by limiting the bandwidth and by proper filtering. Considering the superiority of the digital filters, this paper aims to investigate the performances that could be achieved by a DSP VLC receiver. For this purpose, a new architecture of VLC receiver based on DSP is proposed. In order to enhance the efficiency of the proposed receiver, some of the key blocks are investigated, so that their performances could be improved. Through numerous simulations, the parameters of the different block are improved, enabling the communication in noisy environments. 


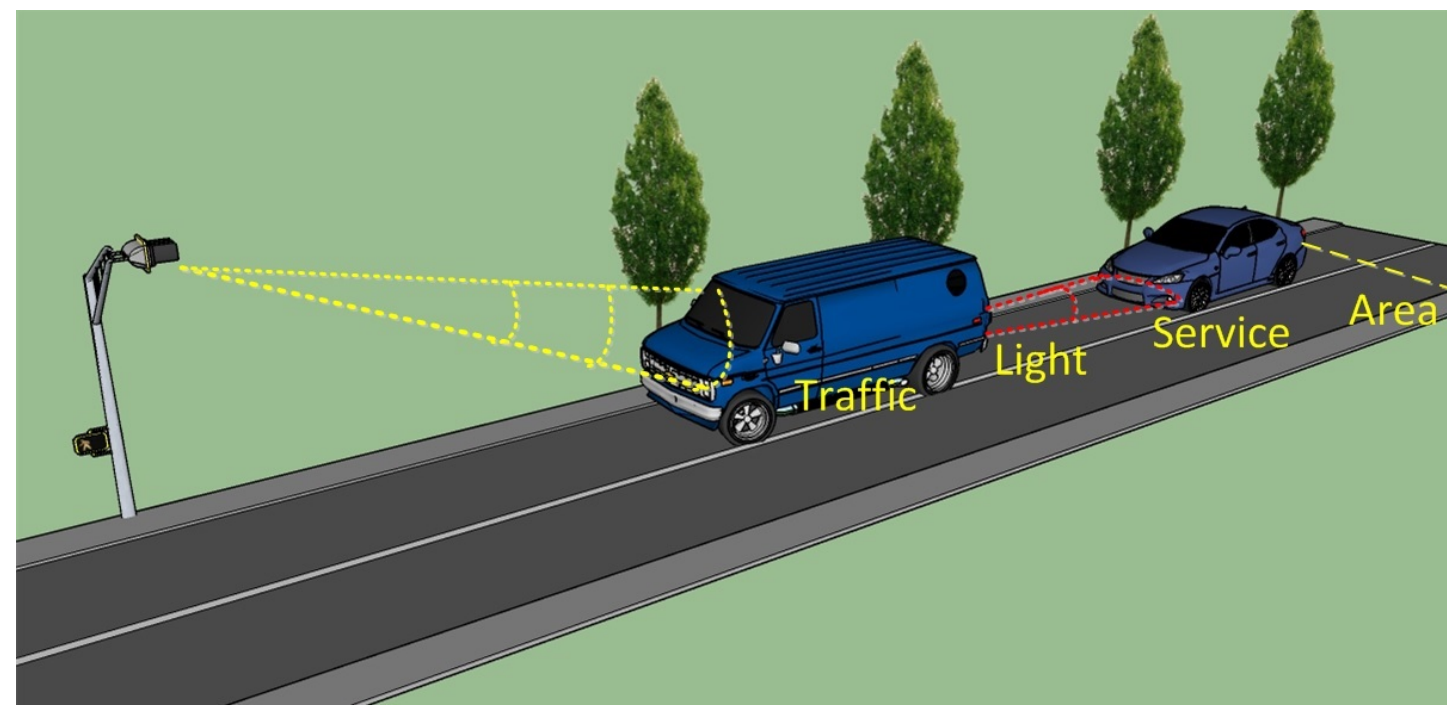

Fig. 1. Illustration of visible light communication between traffic infrastructure and vehicles

\section{CONSIDERATIONS ON THE VLC CHANNEL}

In $\mathrm{VLC}$, the data is modulated onto the instantaneous power of the carrier, technique referred to as Intensity Modulation (IM). For the extraction of the data, the receiver uses the direct detection (DD). In this case, the photodetector, generally a reversed bias silicon photodiode, outputs a photocurrent proportional with the incident light. Following, a transimpedance amplifier converts the small photocurrent into a voltage which is further processed by using analog and/or digital techniques, until the data signal is reconstructed. The transimpedance circuit has low distortion and large gainbandwidth product, representing according to many studies, the best compromise between bandwidth and noise [8, 17]. As a coding technique, the IEEE 802.15.7 standard [18] for wireless optical communication using visible light defines for outdoor low data rate application the usage of the Manchester code, with data rates between 11.67 and $100 \mathrm{~kb} / \mathrm{s}$.

In VLC, the emitter and the receiver are interconnected through the free space optical communication channel. As the visible light is an electromagnetic radiation, similar to all electromagnetic radiations, its intensity decreases proportionally to the square root of the distance as it travels through the communication channel, making the signal that arrives at the receiver to be very weak $\left(\mu \mathrm{W} / \mathrm{cm}^{2}\right)$. Moreover, the VLC channel contains numerous sources of optical noise. In daytime, the most important source of noise is the sun. Other sources of noise are represented by VLC transmitters or by any source of light with or without data transmission capabilities. Artificial light switching or the dynamic conditions also make the VLC channel very unpredictable. In the case of outdoor VLC applications, the unpredictability is even greater because of the weather. Water particle from rain, snow, or heavy fog can affect the VLC link by causing scattering of the light containing the data. The multitude of noise sources, together with the weak signals, especially at long distance, significantly affects the SNR in VLC. Another characteristic of the VLC channel comes from the stringent LoS conditions, which limits the multipath propagation. In outdoor VLC, the multipath has a limited effect which is experienced only at short emitter receiver distances [19].

For the VLC receiver, the SNR is affected by two types of noises: shot noise and thermal noise. The shot noise is strictly dependent of the total light incident on the receiver and in the presence of other light sources, is the dominant noise. The thermal noise is introduced by the preamplifier circuit. In daylight conditions its value is very low compared with the one of the shot noise. However, in the absence of other light sources, it represents the dominant noise. Both shot noise and thermal noise are signal independent and white, and can be modeled as Additive White Gaussian Noise (AWGN). In the context in which the AWGN is dominant in VLC, the following simulations will focus on its effect on the communication. However, other forms of noises may affect VLC.

\section{The Configuration Of The Proposed VLC RECEIVER}

For most of the existing outdoor VLC prototypes, the output of the transimpedance circuit is processed mostly by using analog techniques (see [7 - 10]). Even if this approach has the advantage of a lower implementation cost, future VLC prototypes could take advantage of the use of DSP techniques. The central element of a DSP system is the digital filter. The digital filters can achieve far better results compared with the analogical ones, fact that had a key contribution at the increasing popularity of the DSP systems. Since the outdoor VLC channel is strongly affected by noise, the superiority of the digital filters represents a major advantage that can enhance the performances of future VLC receivers. In order to investigate the performances of such a system, a VLC DSP receiver architecture is proposed and presented in Fig. 2. For the proposed receiver and for the following tests, a modulation frequency $\mathrm{F}=11.67 \mathrm{kHz}$ is considered. All the tests were performed on Manchester encoded messages. As illustrated in 


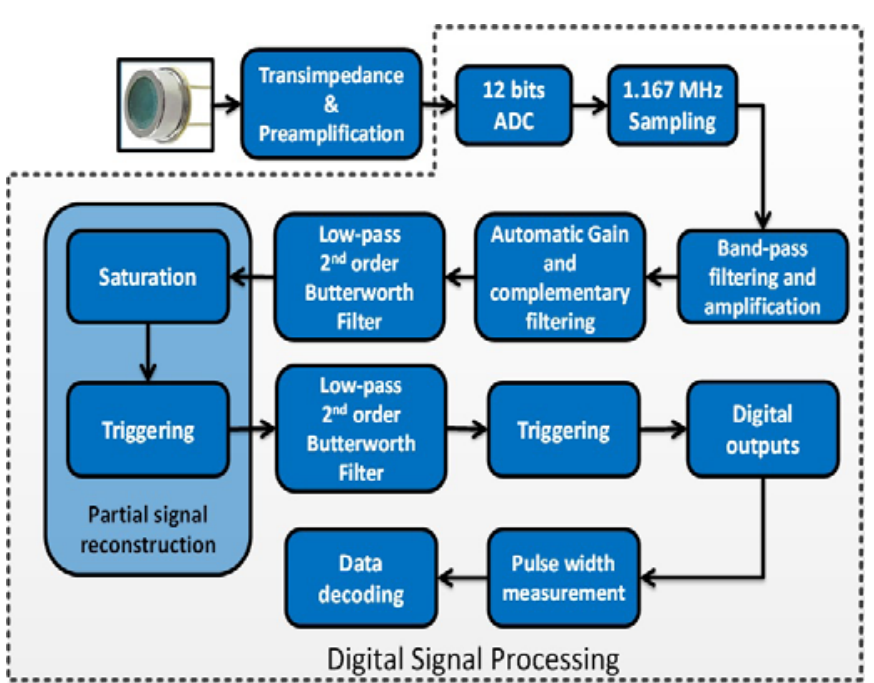

Fig. 2. The architecture of the proposed VLC receiver.
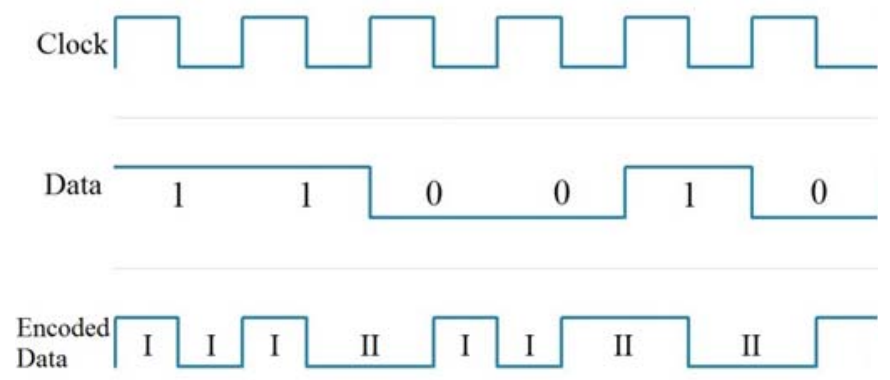

Fig. 3. Data encoding using the Manchester code.

Fig. 3, the Manchester encoding leads to two types of pulses: one that has the period equal with half of the bit period, corresponding with the clock rate of the modulation frequency and one that has twice this period. The effect of the noise and of the filters on the two types of pulses is different. The following results will contain the average for an equal number of pulses from each category. The analog to digital conversion is performed by a 12 bits ADC unit at a sampling frequency of $1.167 \mathrm{MHz}$. However, the performances of the proposed receiver can be greatly improved by using a higher sampling frequency which has as main advantage a better filtering. This fact is illustrated in Fig. 4, where it can be observed how does the sampling frequency influences the pulse distortion in the case of a $2^{\text {nd }}$ order Butterworth filter with a cutoff frequency of $1.5 \cdot \mathrm{F}$, with respect to the SNR.

One of the most important components of a DSP system is the digital filter. The digital filters are the central element of the DSP system and for this reason it is very important to determine the suitable filter and its parameters. Selecting the order of a digital filter represents a tradeoff between the quality of the filtering on one side and the number of mathematical operations performed for each sample on the other side. A higher filter order will provide a better output with lower distortions but will require more computational resources which will increase the cost of the system. Fig. 5 illustrates the manner in which the order influences the quality of the filtering for the case of the Butterworth filter. It can be observed that starting with the $2^{\text {nd }}$ order, the filters have comparable

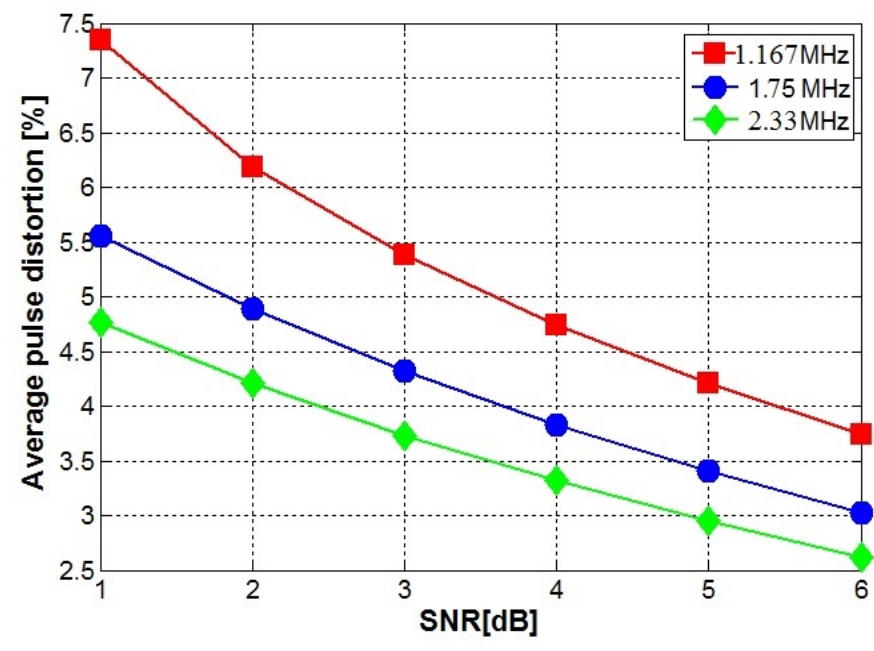

Fig. 4. The influence of the sampling frequency on the filtering quality.

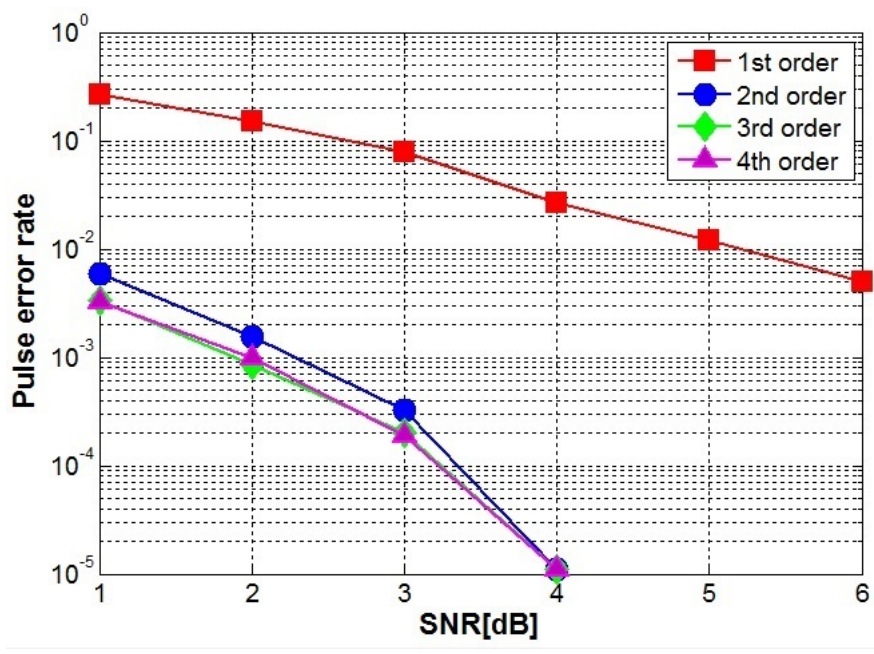

Fig. 5. The influence of the filter order on the filtering quality.

performances in terms of pulse error rate (PER). Based on these results and aiming not to increase the cost of the receiver, the $2^{\text {nd }}$ order filter was considered as a fair trade between performances and resource requirement.

The next investigation aims to confirm the selection of the Butterworth filter by comparing it with the Elliptic and the Chebyshev filters. In other to evaluate the performances of the three filters the pulse length of the reconstructed pulse was measured and distortions of the pulses were determined. The pulse width distortions for the three types of filters are illustrated in Fig. 6. The results confirmed the superior performances of the Butterworth filter.

After selecting the Butterworth filter, the next tests related with the filtering had as purpose to determine the optimal cutoff frequency. In this case, several cutoff coefficients had been chosen and their efficiency has been tested for the proposed receiver. Fig. 7 shows how the different cutoff coefficients affect the PER. Therefore, a cutoff frequency of $1.5 \cdot \mathrm{F}$ was considered. 


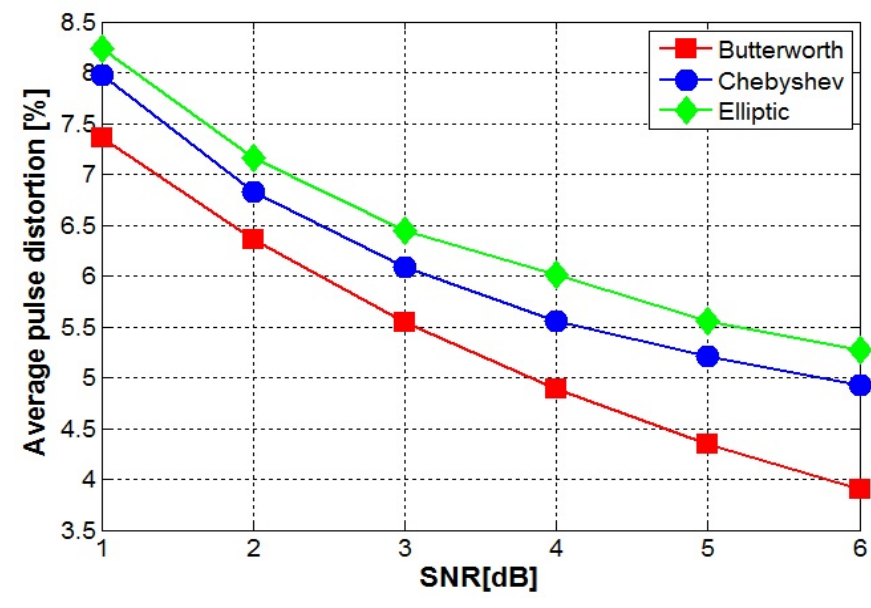

Fig. 6. Pulse width distortion for the Butterworth, Chebyshev and Elliptic filters

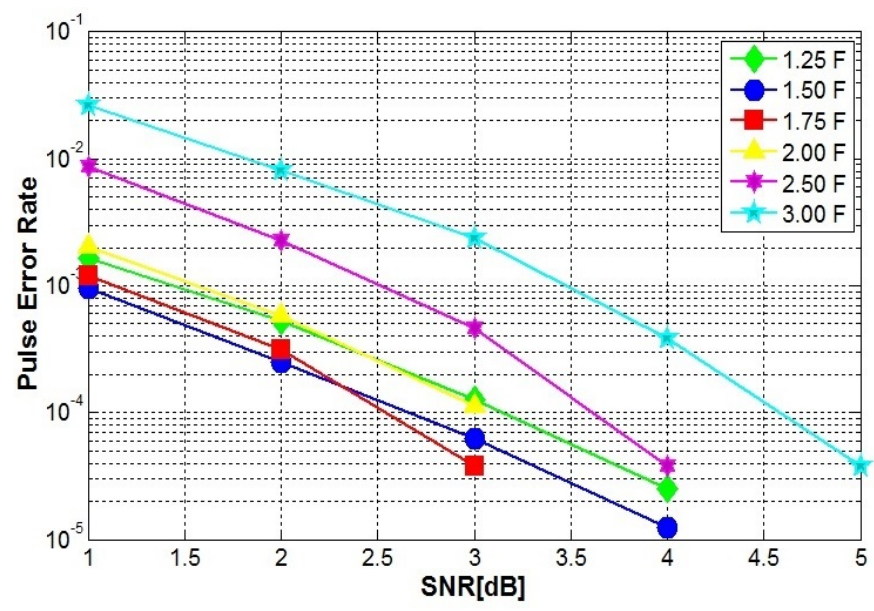

Fig. 7. The influence of the cutoff frequency on the pulse error rate.

Considering the square pulse reconstruction, it is made based on triggering, according with the values of the thresholds. At this level two approaches were considered and investigated: one based on symmetric triggering and one based on asymmetric triggering. For the symmetric approach, the threshold is set at the same value for both the rising and the falling edges, in this case half the data signal's amplitude. For the asymmetric approach, the thresholds for the rising and for the falling edges have different values. The employment of asymmetric thresholds seemed an adequate option because in some cases, the noise leads to the occurrence of peeks that can reach amplitude levels that can go as high as half the useful signal amplitude, or even above. In these cases, by increasing the rising edge threshold prevents false triggering. To compensate the effect of this increase, the threshold of the falling edge must be symmetrically decreased. Under these circumstances, two asymmetric thresholds were investigated: 0.6 and 0.4 respectively 0.65 and 0.35 the signal amplitude. Even if in some specific cases this approach was found useful, its usage has not been found to improve the overall performances. As showed in Fig. 8, the results for these tests showed that the symmetric signal reconstruction had better results in terms of PER.

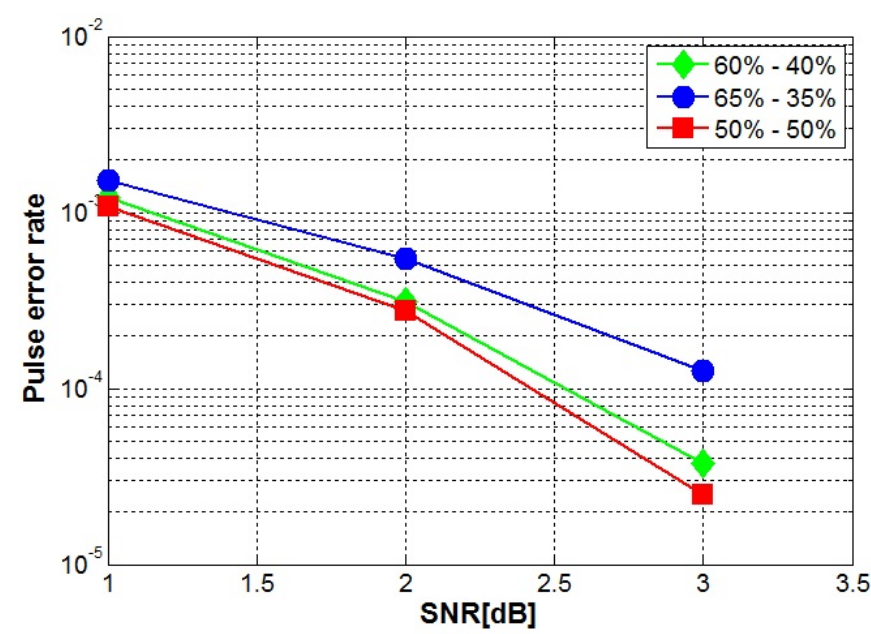

Fig. 8. Pulse error rate for different thresholds

Furthermore, in order to enhance the systems performances, the pulse reconstruction is a progressive one, that uses a partial reconstruction block before the final triggering. This block uses a multi-level triggering based on an adaptive threshold computing algorithm. This way, the threshold is continuously changing its value, within certain limits, based on the input samples and on the values of the previous samples. For every pulse, the values of the previous samples are used to determine the signal minim and the maxim, values that will be used for the threshold computation. Moreover, to help prevent false triggering, the values of the thresholds are modifying their values according with the input signal. An example that illustrates the necessity of the adaptive triggering is illustrated in Fig. 9.a. It can be observed that after the input signal got above the threshold limit the triggering was effectuated, but immediately after, because of the noise, the signal decreased below the threshold triggering a ' 0 ', and then again the signal began to rise, triggering a ' 1 '. To prevent such false triggering, right after the threshold limit is exceeded, the value of the threshold is set to the lowest value, value that is increasing gradually as the input signal amplitude is rising. The effectiveness of this approach is illustrated in Fig. 9.b. Also, it can be observed that the output signal (red) is not a perfectly square signal but a partially reconstructed signal, as an intermediate step towards the square signal triggering.

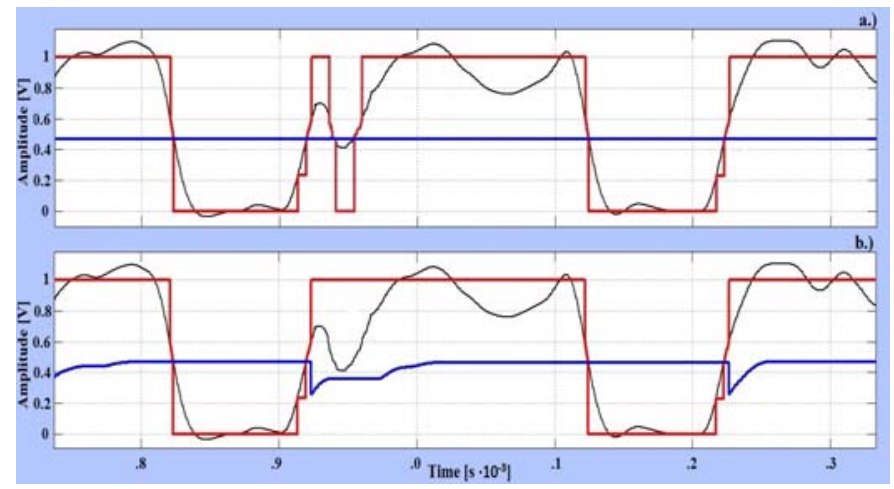

Fig. 9. False triggering prevention using adaptive thresholds; the threshold (blue) is computed based on the current and the previous values of the input signal (black). 


\section{Final Simulation Results}

The final tests were performed in order to determine the Bit Error Ratio (BER) and the Frame Error Ratio (FER) results for the proposed receiver architecture. A digital frame has been defined, as illustrated in Fig. 10. The frame consists of several synchronization bits, a start bit, the data bits and a stop bit. For these tests, short messages of 64 data bits (8 ASCII characters) were sent in different noise conditions. The results for these tests are presented in Fig. 11. It can be observed how the noise affects the BER and the FER results. The results show that for a SNR above $4 \mathrm{~dB}$, the BER is higher than $10^{-5}$. Since the results were obtained without any error correction technique, it can be considered that the proposed receiver architecture is suitable for outdoor VLC. The usage of the Convolutional and of the Reed Solomon codes will further improve the receiver performances.

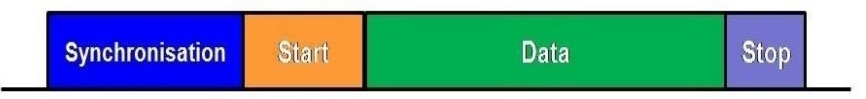

Fig. 10. Structure of the digital frame.

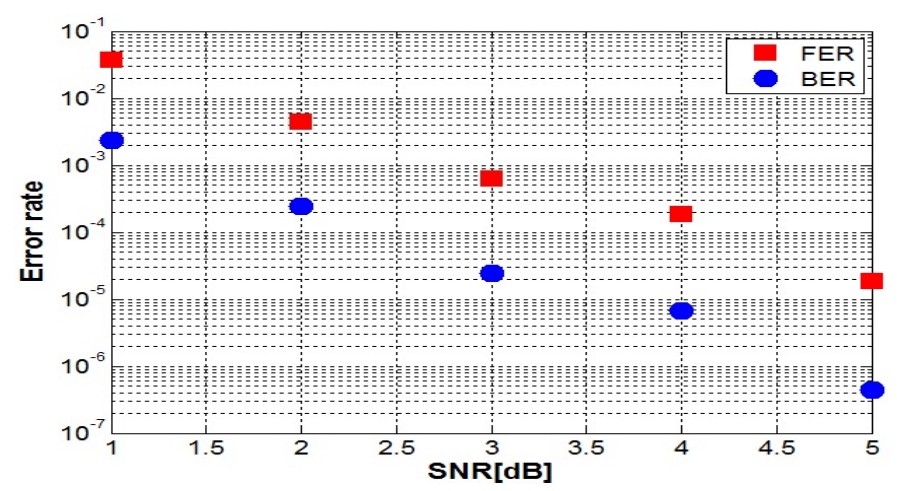

Fig. 11. Frame and bit error rate.

\section{CONCLUSIONS}

The superiority of the digital filters has the potential to enhance the performances of the future VLC receivers. This paper proposed a new DSP VLC receiver architecture and presented an analysis over the aspects that influence its performances. The results of these analyses allowed the selection of the optimal parameters for the proposed architecture. The simulation results confirmed the suitability of the proposed receiver for VLC even at low SNR levels.

Preliminary tests of the architecture were performed on a low cost hardware system (16 bits DSPic) and confirmed the performances. However, due to the high complexity, the system was not fast enough in order to allow for real-time processing.

\section{REFERENCES}

[1] D. Tsonev, Chun Hyunchae, S. Rajbhandari, J.J.D. McKendry, S. Videv, E. Gu, M. Haji, S. Watson, A.E. Kelly, G. Faulkner, M.D. Dawson, H. Haas, D. O'Brien, "A 3-Gb/s Single-LED OFDM-Based Wireless VLC Link Using a Gallium Nitride $\mu$ LED," Photonics Technology Letters, IEEE , vol.26, no.7, pp.637,640, April1, 2014.
[2] Yew-Kiat Cheong; Xiao-Wei Ng; Wan-Young Chung, "Hazardless Biomedical Sensing Data Transmission Using VLC," Sensors Journal, IEEE , vol.13, no.9, pp.3347,3348, Sept. 2013.

[3] I.L. Azevedo, M.G. Morgan, F. Morgan, "The Transition to Solid-State Lighting," Proceedings of the IEEE, vol.97, no.3, pp.481,510, 2009.

[4] M.S. Shur, A. Zukauskas, "Solid-State Lighting: Toward Superior Illumination," Proceedings of the IEEE, vol.93, no.10, pp.1691,1703, Oct. 2005.

[5] A. Lavric, V. Popa, I. Finis, "The design of a street lighting monitoring and control system," Electrical and Power Engineering (EPE), 2012 International Conference and Exposition on , , pp.314,317, 2012.

[6] I. Takai, S. Ito, K. Yasutomi, K. Kagawa, M. Andoh, S. Kawahito, "LED and CMOS Image Sensor Based Optical Wireless Communication System for Automotive Applications," Photonics Journal, IEEE, vol.5, no.5, Oct. 2013

[7] A. Cailean, B. Cagneau, L. Chassagne, S. Topsu, Y. Alayli, J-M. Blosseville, "Visible light communications: Application to cooperation between vehicles and road infrastructures," Intelligent Vehicles Symposium (IV), 2012 IEEE , pp.1055,1059, 3-7 June 2012.

[8] N. Kumar, D. Terra, N. Lourenco, L.N. Alves, Rui L. Aguiar, "Visible light communication for intelligent transportation in road safety applications," Wireless Communications and Mobile Computing Conference (IWCMC), 2011 7th International, pp.1513,1518, 2011.

[9] A. Cailean, B. Cagneau, L. Chassagne, S. Topsu, Y. Alayli, M. Dimian, "A robust system for visible light communication," Wireless Vehicular Communications (WiVeC), 2013 IEEE 5th International Symposium on , vol., no., pp.1,5, 2-3 June 2013.

[10] S. Okada, T. Yendo, T. Yamazato, T.Fujii, M. Tanimoto, Y. Kimura, "On-vehicle receiver for distant visible light road-to-vehicle communication," Intelligent Vehicles Symposium, 2009 IEEE , vol., no., pp.1033,1038, 3-5 June 2009.

[11] T. Nagura, T. Yamazato, M. Katayama, T.Yendo, T. Fujii, H. Okada, "Improved Decoding Methods of Visible Light Communication System for ITS Using LED Array and High-Speed Camera," Vehicular Technology Conference (VTC 2010-Spring), 2010 IEEE 71st , vol., no., pp.1,5, 16-19 May 2010.

[12] A.-M. Cailean, B. Cagneau, L. Chassagne, S. Topsu, Y. Alayli, M. Dimian, "Visible light communications cooperative architecture for the intelligent transportation system," Communications and Vehicular Technology in the Benelux (SCVT), 2013 IEEE 20th Symposium on , vol., no., pp.1,5, 21-21 Nov. 2013.

[13] IEEE Standard for Information technology-- Local and metropolitan area networks-- Specific requirements-- Part 11: Wireless LAN Medium Access Control (MAC) and Physical Layer (PHY) Specifications Amendment 6: Wireless Access in Vehicular Environments," IEEE Std 802.11p-2010, vol., no., pp.1,51, July 152010.

[14] A.-M. Cailean, B. Cagneau, L. Chassagne, V. Popa, M. Dimian, "A survey on the usage of DSRC and VLC in communication-based vehicle safety applications," Communications and Vehicular Technology in the Benelux (SCVT), 2014 IEEE 21st Symposium on , 10 Nov. 2014.

[15] S. Eichler, "Performance Evaluation of the IEEE 802.11p WAVE Communication Standard", In IEEE Vehicular Technology Conference, 2007.

[16] K. Bilstrup, E. Uhlemann, E. Strm, U. Bilstrup, "On the ability of the 802.11p MAC method and STDMA to support real-time vehicle-tovehicle communication," EURASIP Journal on Wireless Communications and Networking 2009, 2009:902414.

[17] J.M. Kahn, J.R. Barry, "Wireless infrared communications," Proceedings of the IEEE, vol.85, no.2, pp.265,298, Feb 1997.

[18] IEEE Standard for Local and Metropolitan Area Networks--Part 15.7: Short-Range Wireless Optical Communication Using Visible Light, IEEE Standard, 2011, 1-309.

[19] C. Liu, B. Sadeghi, E. W. Knightly, "Enabling vehicular visible light communication (V2LC) networks," Proceedings of the Eighth ACM international workshop on Vehicular inter-networking (VANET '11), ACM, New York, NY, USA, 41-50, 2011. 